\title{
Experimental and Numerical Research on Flow-Induced Vibration in Valves
}

\author{
Asmaa Ali Hussein \\ Foundation of Technical Education/ Baghdad- Iraq \\ Email: asmaa31930@yahoo.com
}

(Received 4 September 2016; accepted 3 January 2017)

https://doi.org/10.22153/kej.2017.01.001

\begin{abstract}
All central air conditioning systems contain piping system with various components, sizes, material, and layouts. If such systems in operating mode, the flow in piping system and its component such as valves can produce severe vibration due to some flow phenomenon's. In this research, experimental measurements and numerical simulation are used to study the flow-induced vibration in valves. Computational fluid dynamics (CFD) concepts are included with one-way and two-way fluid-structure interaction concepts by using finite element software Package (ANSYS 14.57). Detection analysis is performed on flow characteristics under operation conditions and relations with structural vibration. Most of real geometrical, operational, and boundary conditions are simulated to obtain best similarity with real operation conditions. Comparisons performed between experimental data and numerical results (one-way and twoway simulation) to verify the results. The main conclusion was drawn from the study that the dominant source of vibration for valve is the water pulsation in addition to amount of water hammering. In addition, the main source of water pulsation in globe valve is the vortex shedding and pressure difference between upstream and downstream of valve. The vibration amplitude was increased with increasing flow rate until to be maximum when the flow rate around $30 \%$ and then decreased until flow rate reaches to around $85 \%$ and then trends to be constant.
\end{abstract}

Keywords: Flow-induced vibration, vortex shedding, pressure pulsation, valve, finite element, ANSYS, fast Fourier transform (FFT).

\section{Introduction}

The vibration in piping system and its component especially valves can occurs due to many reasons. Some of these reasons related to unavoidable flow phenomenon such as turbulence, cavitation, vortex shedding, and pressure pulsation. The other reasons related to structural behavior such as the resonance between the long pipeline and the other vibrating equipment such as pumps. The high level of vibration in piping system can cause breakdowns because the leaks, noise, fracture, and fatigue failure. The reduction of vibration level in such systems is a valuable task to avoid breakdown. The reduction of vibration requires a wide knowledge of vibration source, causes, types, and how can to eliminate or reduce the causes. The prior investigation of flow behavior and flow-induced vibration of such system will give a most important knowledge for optimum anti vibration design. The valve is one of the main components of any piping system. The behavior of flow inside the valve and interaction between the flow and valve structure components can be the source of severe vibration. N. H. Fletcher [1] introduced a theoretical study on the oscillations of piping fittings. The main conclusion from this study is that there exist typical ranges of acoustic resistance for oscillation is possible. The analysis process the formation of oscillations of little amplitude. Lighterink et. al [2] presented a paper described a method to examine the loads on tools due to the mechanical vibrations. Investigations performed on a piping system with standard equipment to investigate: a) the vibrational natural mode shape of the piping, 
b) the natural mode shapes of the piping and equipment, and c) the total loads on the equipment. Ravi Pankhania et. Al [3] carried out study on vibration of piping equipment for nuclear application. The study included design and analysis of motor operated flow control used for nuclear application. Malik I. Al- Amayreh et.al [4] presented a research on butterfly valve for vibration reduction. The work included numerical simulation of a butterfly valve used to control the flow rate in industrial process. Present research includes experimental and numerical investigation on flow-induced vibration in globe valve.

\section{Numerical Simulation}

Computation fluid dynamics (CFD) with the aid of finite element software package (ANSYS 14.57)is used in the current study. This program analyzes and solves a wide range of different mechanical problems, such as the structural, fluid flow, thermal, and the fluid-structure interaction problems [5]. ANSYS mechanical (APDL 14.57)is used for structural analysis and ANSYS (Fluent 14.57) is used for fluid analysis and ANSYS workbench is used for fluid-structure interaction analysis. CFDisused to investigate the flow characteristics for the vicinity of valve. The flow characteristics included pressure and velocity distribution, velocity vector, vortex, cavitation regions, and the integrated dynamic forces on the structure. FSI applications involve coupling of fluid dynamics and structure mechanics disciplines: 1) Fluid flow exerts hydrodynamic forces on a structure and deforms and/or translates the structure. 2) Deformed or translated structure impart displacement to the fluid domain and changes its shape and thus changes the fluid flow. There are two modes of FSI. One-way solution used with conditions of very small of deformations in the structure. The solver calculates and passes flow fields from CFD to the structural analysis and no need to update and recalculate flow. Two-way is used when Large structural deformations is included where iterate between fluid solution and structural solution until the convergence is obtained. Both 1-way and 2way fluid-structure simulation are used in current research. The coupling of two- way solution is shown in Figure (1) [6].The aims of using the two simulations is to establish the accurate and best simulation for the problem and specify which mode of FSI give results close to experimental results. The numerical simulation will give good visualization of flow behavior inside the valves. Numerical simulation setup requires choosing the perfect geometry representation, suitable element type, boundary conditions, and material properties.

\subsection{Element Type}

Two types of element used in current research. For structural analysis, SHELL281is used. The element has eight nodes with six degrees of freedom at each node. For fluid analysis, higher order tetrahedral element (10 node) with hanging nodes is used. Figure (2) shows the elements type.

\subsection{Boundary Condition}

For structural analysis, clamped boundary (all degrees of freedom $=0$ ) used at the beginning and the end of valves to simulate the flanged connection of valves with piping system. For fluid analysis, velocity inlet boundary $(\mathrm{V}=1 \mathrm{~m} / \mathrm{s})$ used at the inlet of valve and wall boundary $(\mathrm{V}=0)$ used to bound fluid and solid contact.

\subsection{Material Properties}

The fluid used as flow material is water at average temperature $15^{\circ} \mathrm{c}$. The standard values of density and viscosity of water at this temperature used in numerical simulation. The material of valve body is cast iron. The standard values of mechanical properties (density, modulus of elasticity, and poissons ratio) according to ASTM for cast iron are used in structural analysis.

\subsection{Simulation Setup}

The one-way simulation included the transient fluid flow solution for time history of pressure distribution and then export the pressure solution at each time step to transient structural solution to obtain the time history of vibrational displacement. The solution results data obtained and then plotted with the aid of suitable graphic program. The two-way simulation included coupling solution of flow and structural solution to obtain the vibrational displacement directly. The fundamental steps associated with the application of the finite element method to solve problems in fluid simulation showed in the diagram in figure (1) [7]. 


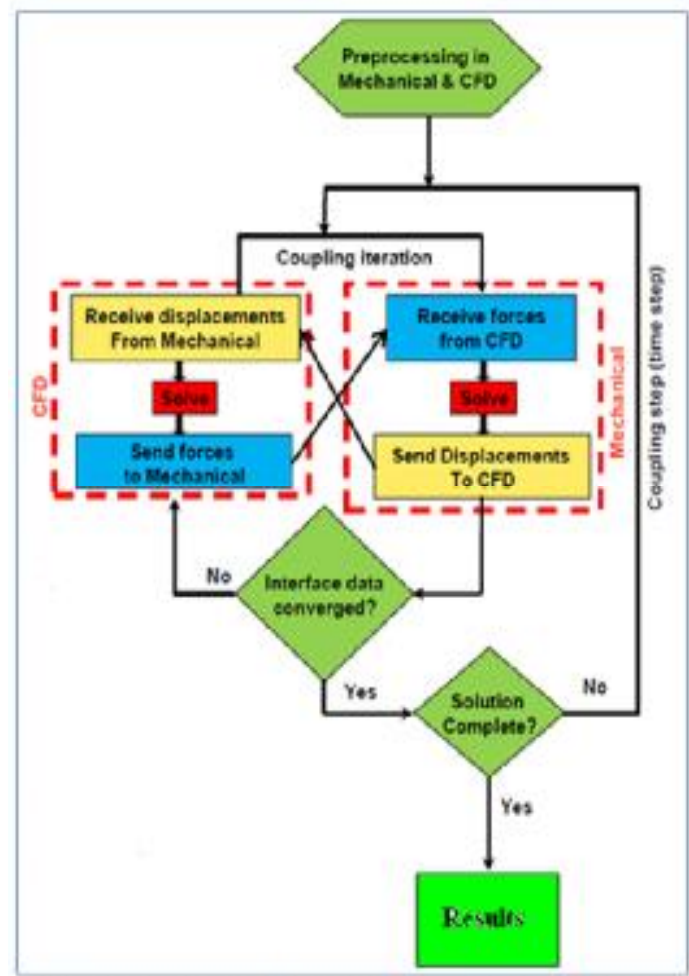

coupling procedure of 2-way fluid-structure interaction [6]

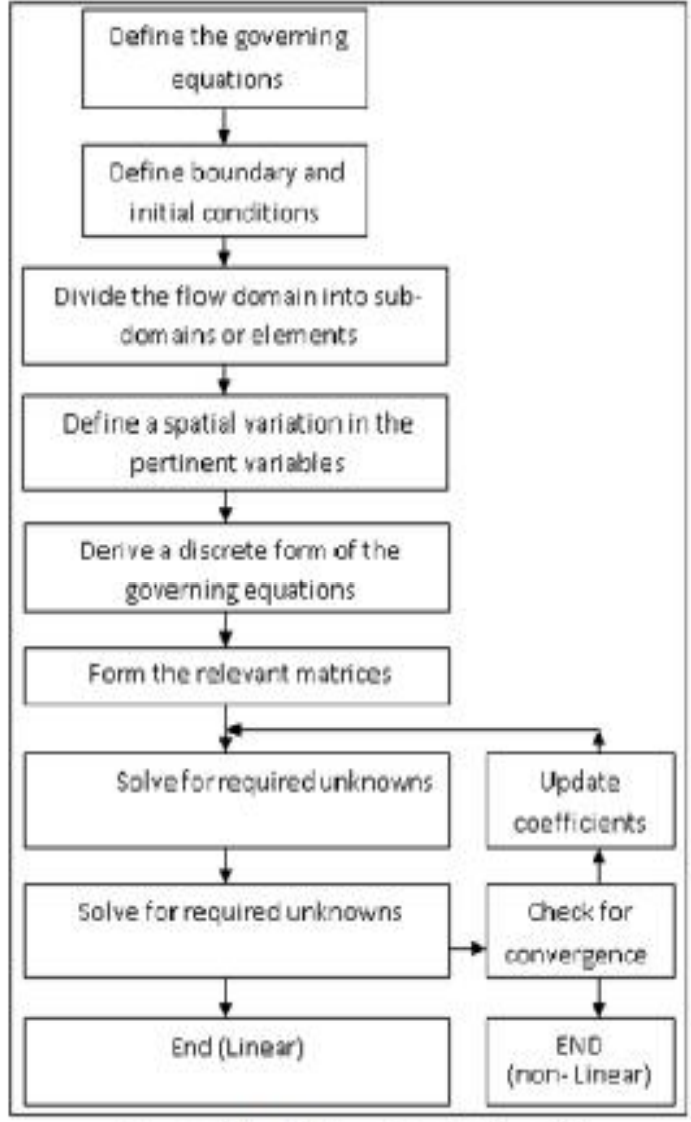

steps of fluid flow simulation [7]

Fig. 1. Coupling procedure and numerical simulation steps.

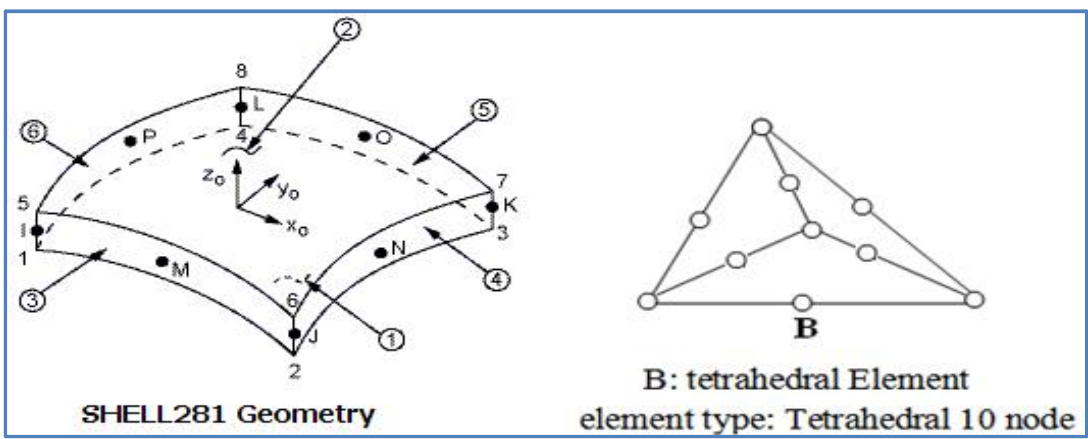

Fig. 2. Element type.

\section{Theoretical background}

\subsection{Flow analysis}

When dealing with water flows, some assumptions included:

1- Three dimensional flow, 2-Incompressible flow, 3-Newtonian fluid.

The governing equations of flow can be summarized by [8]:

(i) Mass conservation (continuity equation)

Equating the quantity of mass entering and leaving an element volume is:

$$
\frac{\partial u}{\partial x}+\frac{\partial v}{\partial y}+\frac{\partial w}{\partial z}=0
$$

Where:

$\mathrm{x}, \mathrm{y}$, and $\mathrm{z}$ are the Cartesian coordinates.

$\mathrm{u}, \mathrm{v}$, and $\mathrm{w}$ are the velocity component in direction of $\mathrm{x}, \mathrm{y}$, and $\mathrm{z}$ respectively $[\mathrm{m} / \mathrm{s}]$.

(ii) Momentum conservation (Navier-Stokes Equations)

Momentum conservation equation is:

- in $x$-direction : 
$\frac{\partial P}{\partial x}+\rho\left(u \frac{\partial u}{\partial x}+v \frac{\partial u}{\partial y}+w \frac{\partial u}{\partial z}\right)=\rho g_{x}+$

$\mu\left(\frac{\partial^{2} u}{\partial x^{2}}+\frac{\partial^{2} u}{\partial y^{2}}+\frac{\partial^{2} u}{\partial z^{2}}\right)$

-in y-direction :

$\frac{\partial P}{\partial y}+\rho\left(u \frac{\partial v}{\partial x}+v \frac{\partial v}{\partial y}+w \frac{\partial v}{\partial z}\right)=\rho g_{y}+$

$\mu\left(\frac{\partial^{2} v}{\partial x^{2}}+\frac{\partial^{2} v}{\partial y^{2}}+\frac{\partial^{2} v}{\partial z^{2}}\right)$

- in $z$-direction :

$\frac{\partial P}{\partial z}+\rho\left(u \frac{\partial w}{\partial x}+v \frac{\partial w}{\partial y}+w \frac{\partial w}{\partial z}\right)=\rho g_{z}+$

$\mu\left(\frac{\partial^{2} w}{\partial x^{2}}+\frac{\partial^{2} w}{\partial y^{2}}+\frac{\partial^{2} w}{\partial z^{2}}\right)$

Where:

P: pressure $\left(\mathrm{N} / \mathrm{m}^{2}\right)$

$\rho$ : fluid density $\left(\mathrm{kg} / \mathrm{m}^{3}\right)$

g: gravitational acceleration $\left(\mathrm{m} / \mathrm{s}^{2}\right)$

$\mu$ : Viscosity (N.s/m $\left.{ }^{2}\right)$

The comprehensive formulation of above equations in finite element analysis detailed in reference [9].

\subsection{Structural Analysis}

The vibration responses of any continous structure due to external load can be evaluated by using numerical technique to solve the equation of motionwhich can be written as [9].

$[M] \overline{\widetilde{\delta}}+[C] \bar{\delta}+[K] \bar{\delta}=\bar{F}(t)$

$\overline{\tilde{\delta}}=[M]^{-1}(\bar{F}(t)-[K] \bar{\delta}-[C] \overline{\dot{\delta}})$

Where:

[M]: Mass matrix [kg]

[c]: damping coeficient matrix $[\mathrm{kg} / \mathrm{s}]$

$[\mathrm{k}]$ : stifness matrix $\left[\mathrm{kg} / \mathrm{s}^{2}\right]$

$\delta, \dot{\delta}, \ddot{\delta}$ : displacement, velocity andacceleration of structural particle respectively.

The complete procedure of numerical solution for above structural equations presented in reference [9].

\section{Experimental Work}

Experimental work included vibration measurements for piping system components. The experimental tests performed on the piping of central air conditioning system in university of Baghdad because this system suffers severe mechanical vibration. The piping system contains many components such as valves, strainers, elbows, tees, and flow meters. The flow rate inside the system can be controlled with the aid of pumps and valves. The vibration measuring system can be used for this purpose. This system included three-axis accelero meter which can be mounted on any position in piping system to measure the dynamic acceleration of vibrational structure. Then, the output acceleration signal goes to Analog to digital convertor (ADC) card and then showed and recorded by using suitable computer software. Figure (3)shows the flow of vibration signal and apparatus of vibration signal measurements used in the current research. The accelerometer attached rigidly to the structure to measure the acceleration at any point in the structure in the form of signal. The more details and calibration of the vibration measuring devices performed and detailed in reference [10].

\section{Description of Piping System}

All experimental tests performed on the piping network of central air conditioning system of the central library in university of Baghdad. The network consists of pipes with different diameters, lengths, and thickness. Also, there are many types of valves such as gate valve, balance valve, globe valve, and check valve. The vibration measurements performed on globe valve of piping network. 


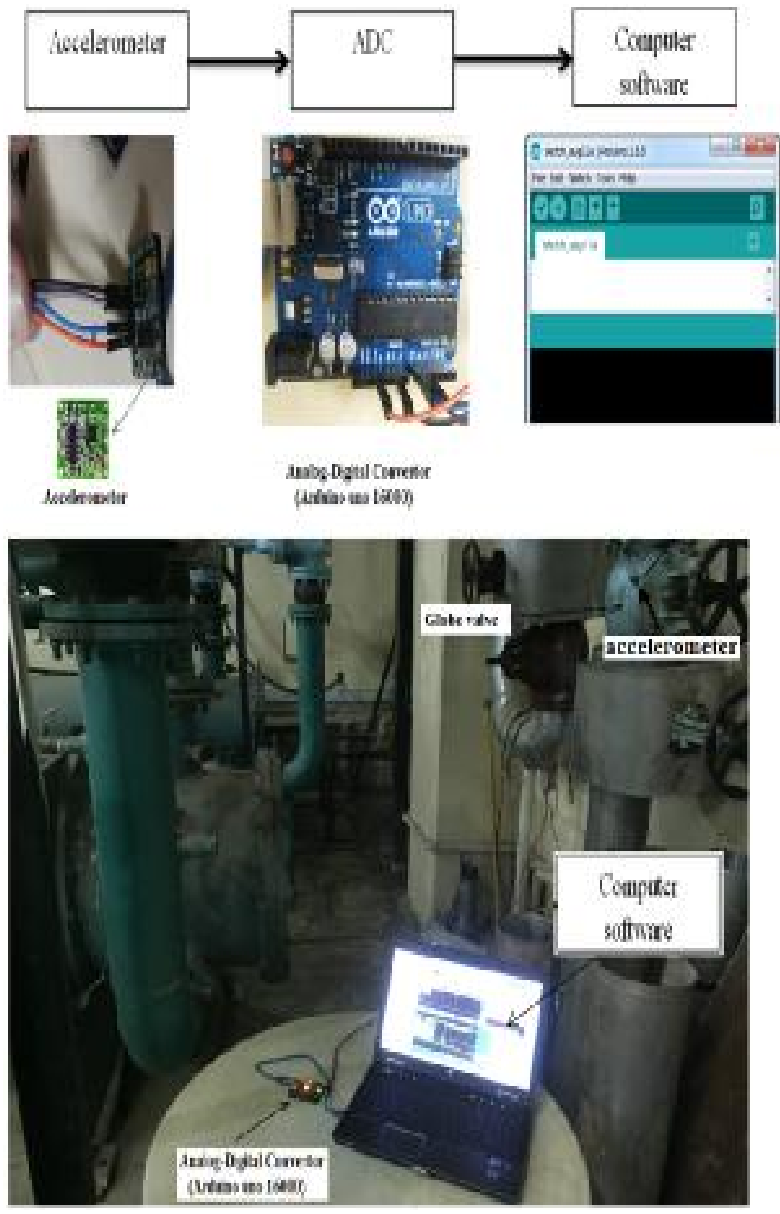

Fig. 3. Flow diagram of vibration signal and vibration measurement devices.

\section{Analysis Procedure}

The most important task is to establish a feasible, precise, and logical analysis for the obtained experimental and numerical data. Precise analysis will lead to precise results and conclusions. In the current research, specific procedureis used to analyze the obtained data. This procedure consists of the following steps:

1. Obtain the experimental data from vibration measurement tests for globe valve. The vibration dataplotted in both time domain and frequency domain.

2. Make suitable and precise numerical simulation for the flow inside the valve, which examined experimentally at step 1

3. From the numerical simulation of step 2, the response of piping system to flow excitation which is the time history of structure displacement (vibration) due to flow dynamic forces can be obtained. These structure displacements (vibration) obtained from both 1-way and 2-way numerical simulations.
4. Make a precise correlation between the numerical results of structure vibration and flow characteristics to find the real reasons of structure vibration.

5. Make precise comparisons between the results of structure vibration obtained from experimental tests and numerical simulations.

6. Make feasible and precise correlation between the experimental results of structure vibration and flow characteristics obtained from numerical simulations

7. Establish the conclusions.

\section{Results and Discussions}

The schematic diagram of the components of globe valve is shown in the figure (4). It mainly consists of two important regions: flow domain which the space occupied by fluid and contains all flow phenomenon's which the main cause of dynamic fluid forces and the pipe wall which represents the structure domain whose vibration is to be measured.
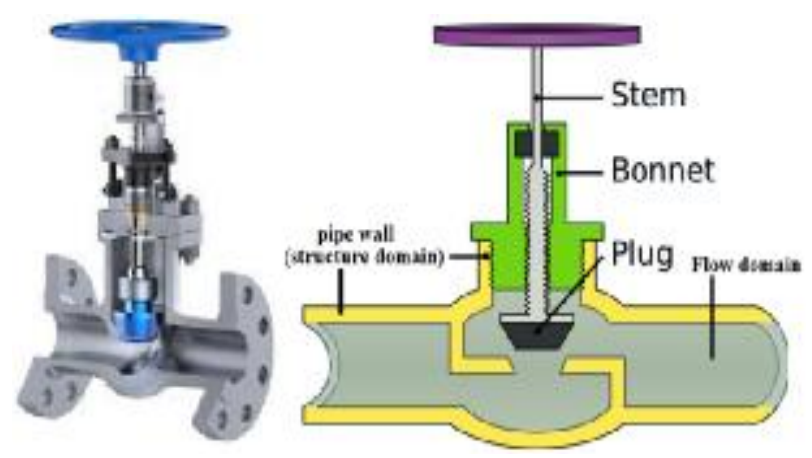

Fig. 4. Schematic diagram of globe valve.

The geometry of the flow domain is an important parameter for initiation and formation of the flow phenomenon. Figure 5 shows the meshed flow domain for numerical simulation at different enclosure ratio. 


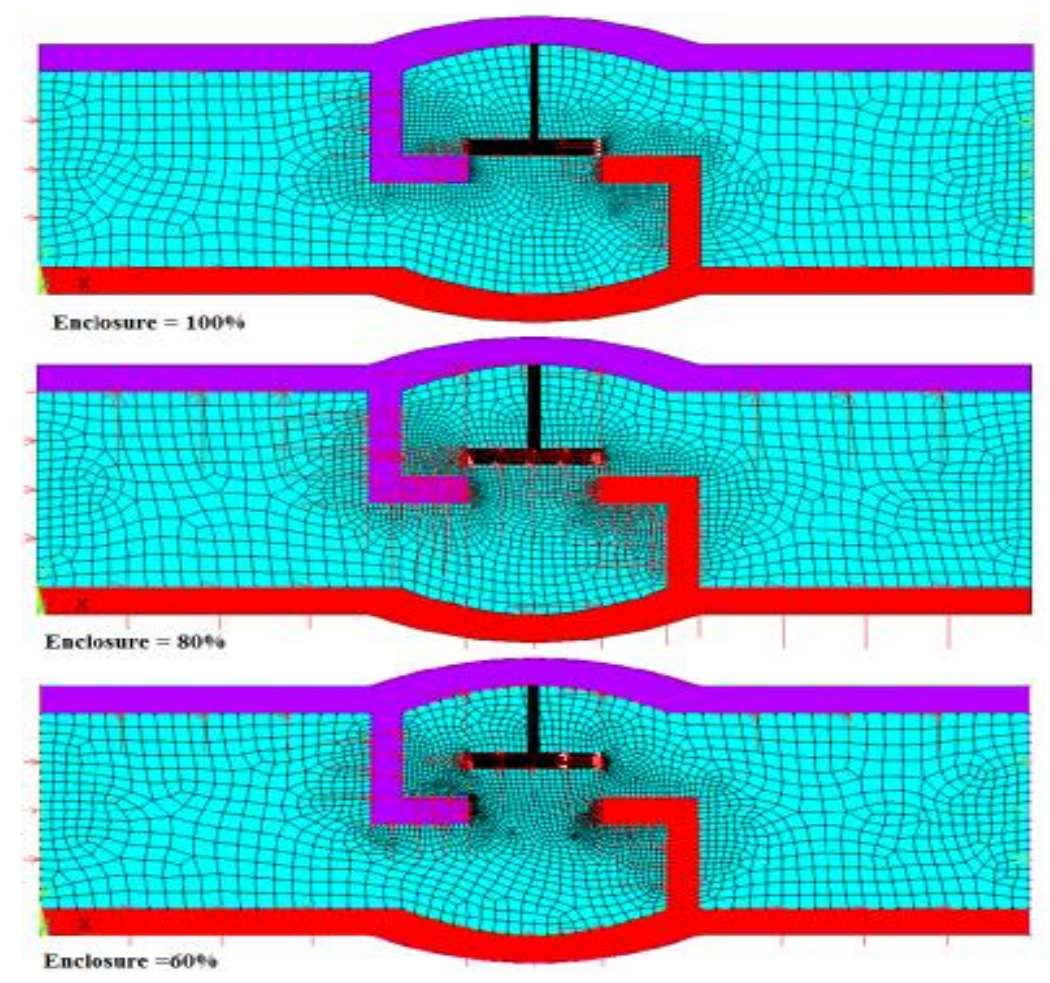

Fig. 5. Flow domain at different enclosure ratio.

Figures6 and 7 show the velocity distribution and pressure distribution obtained from numerical simulation. The distribution of pressure and velocity over the flow domain depends mainly on amount of enclosure of the valve. Figure6 shows the pressure distribution at enclosure 100\%, $80 \%$ and $60 \%$ respectively while figure 7 shows the velocity distribution for the same enclosure ratio respectively. The entrance flow velocity is $1 \mathrm{~m} / \mathrm{s}$.

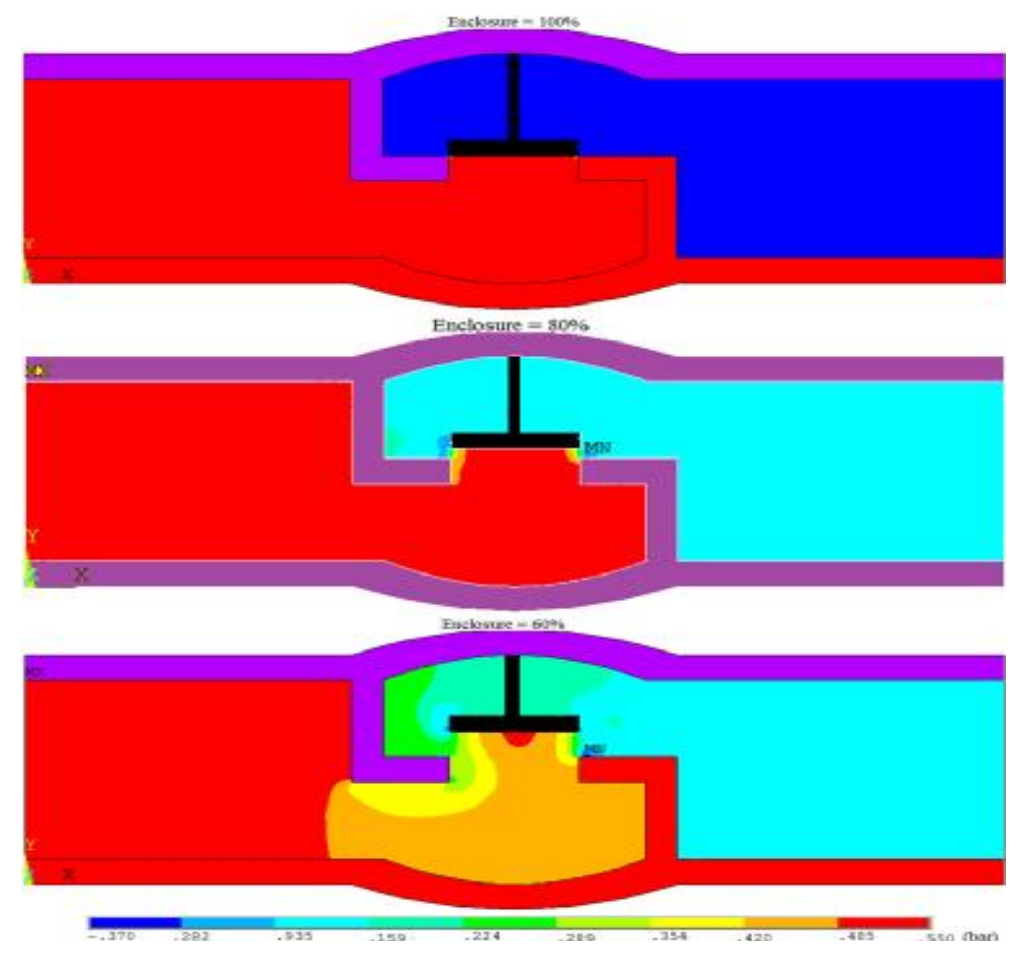

Fig. 6. Pressure distribution over the flow domain of globe valve at different enclosure ratio. 


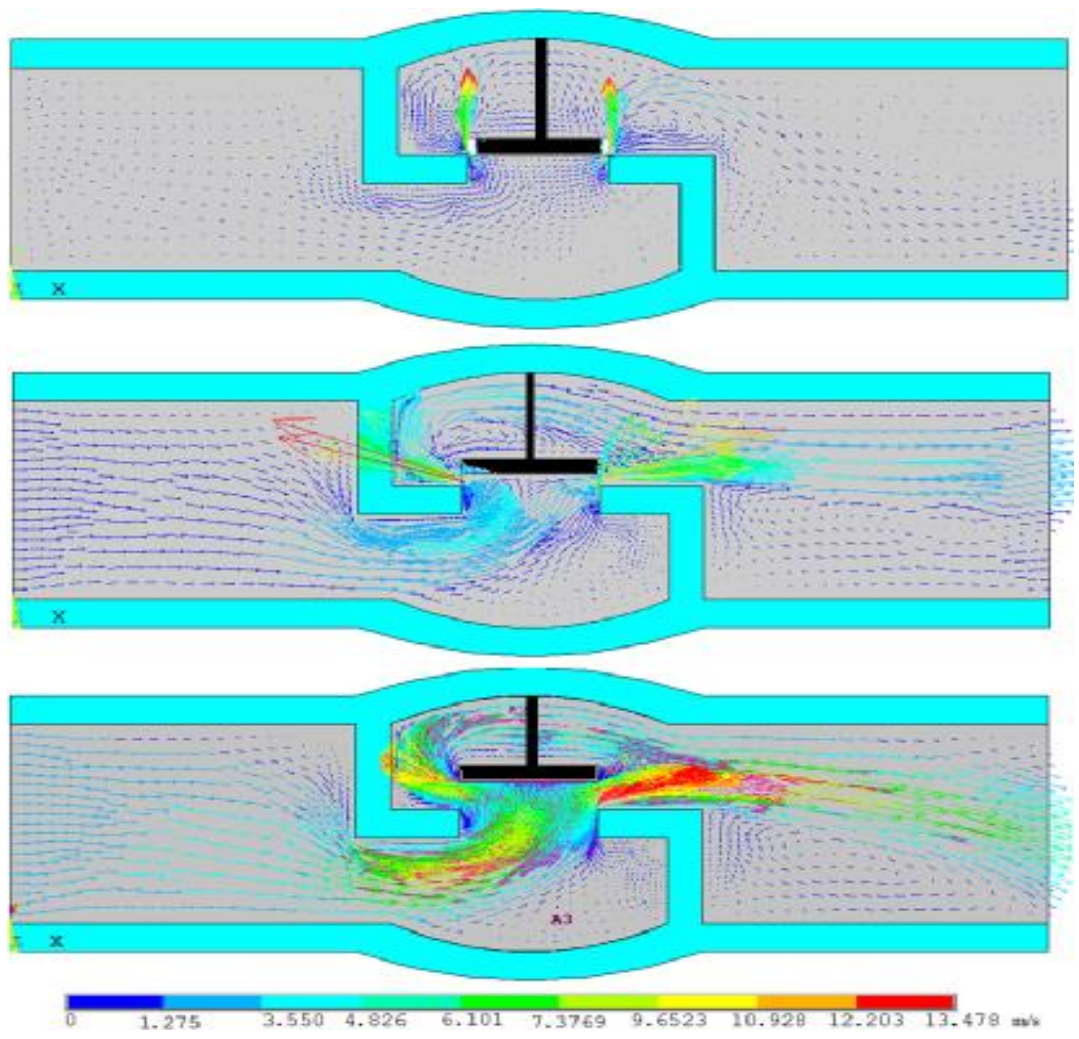

Fig. 7. Velocity vector distribution over the flow domain of globe valve at different enclosure ratio.

Figure 8and 9 show the dynamic response (vibration) on the valve wall obtained from 1-way and 2-way numerical solution respectively in both time and frequency spectrum (FFT). These results obtained from numerical simulation as a table of time history of wall displacement and then drawn by using graphic program.Figure10shows the experimental vibration measurement for the same valve with enclosure of $30 \%$ obtained by experimental test in both time and frequency spectrum (FFT).
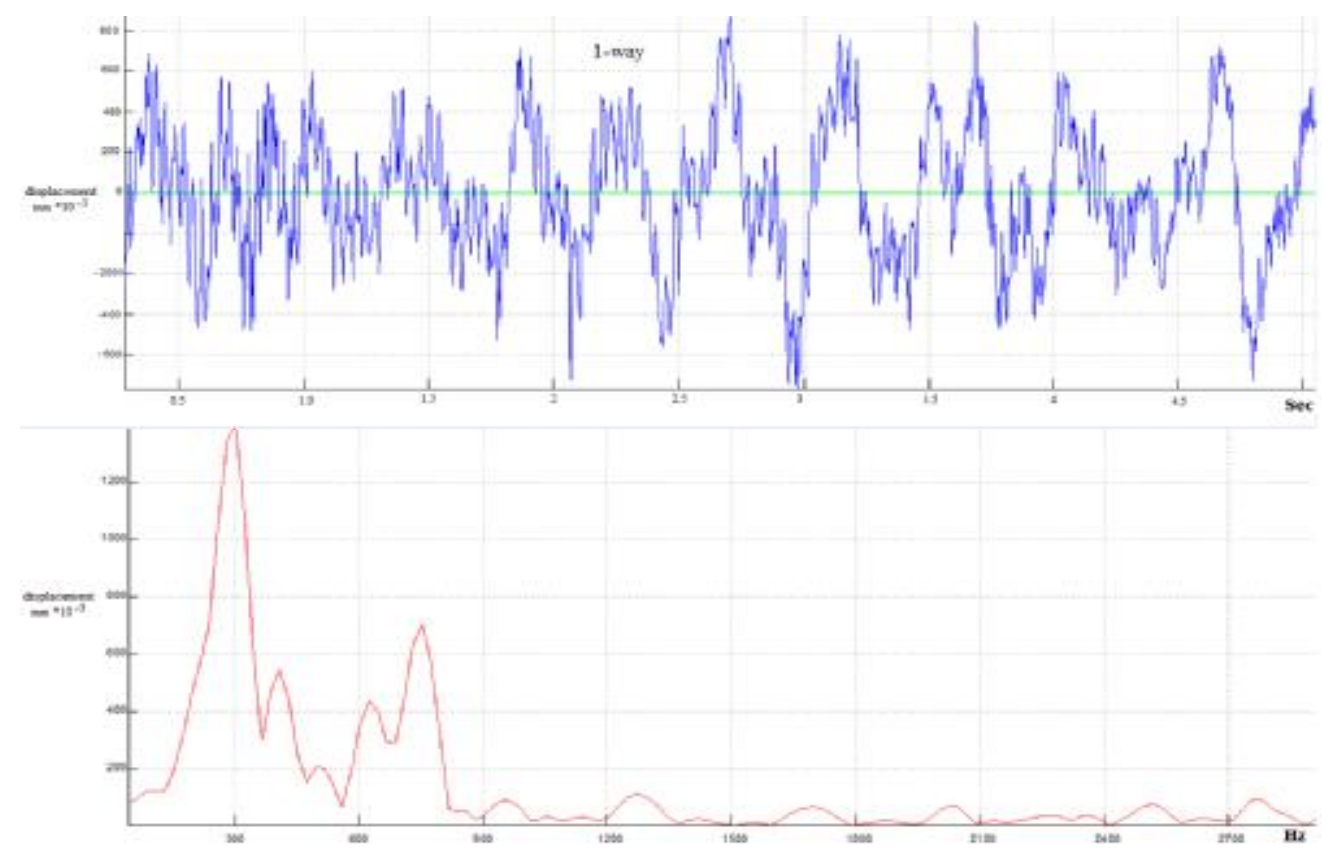

Fig. 8. Displacement of globe valve obtained from numerical solution 1-way. 


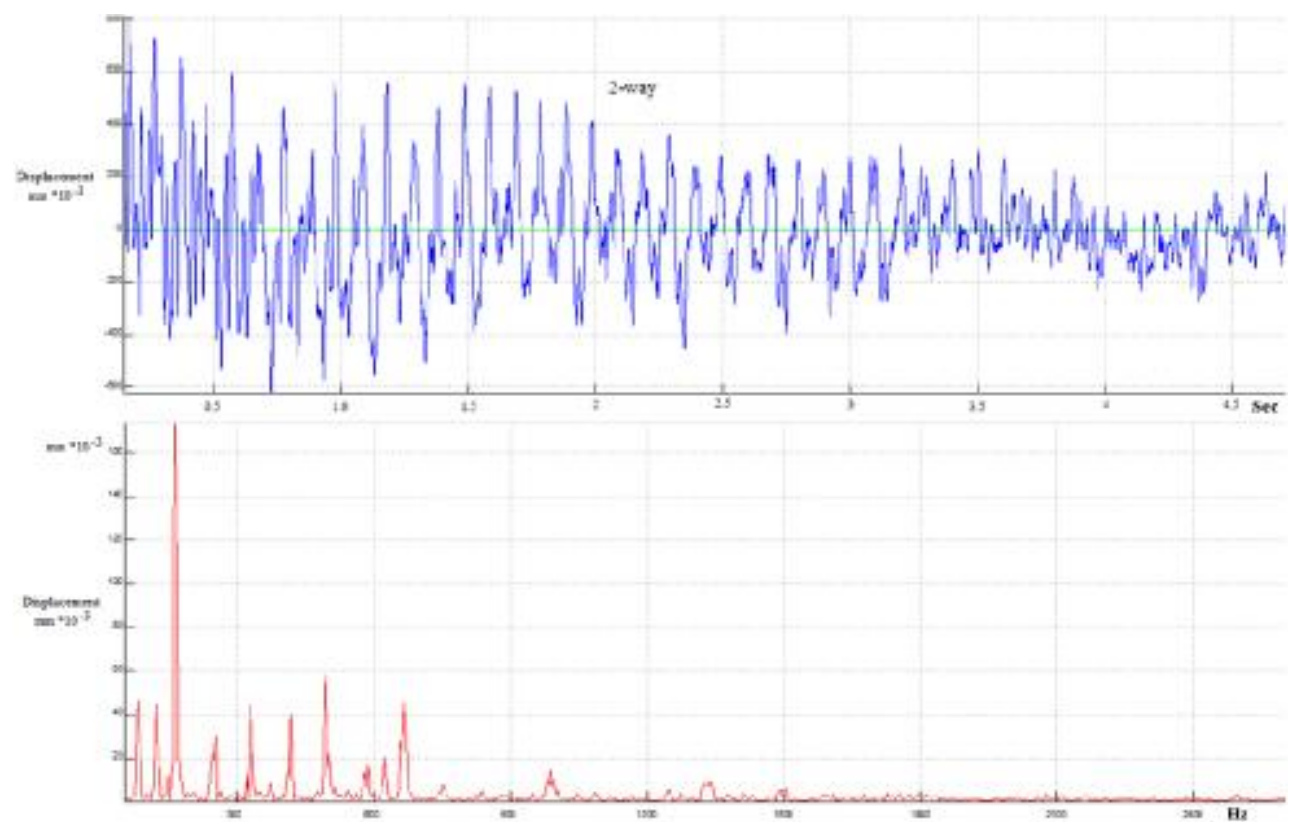

Fig. 9. Displacement of globe valve obtained from numerical solution 2-way.

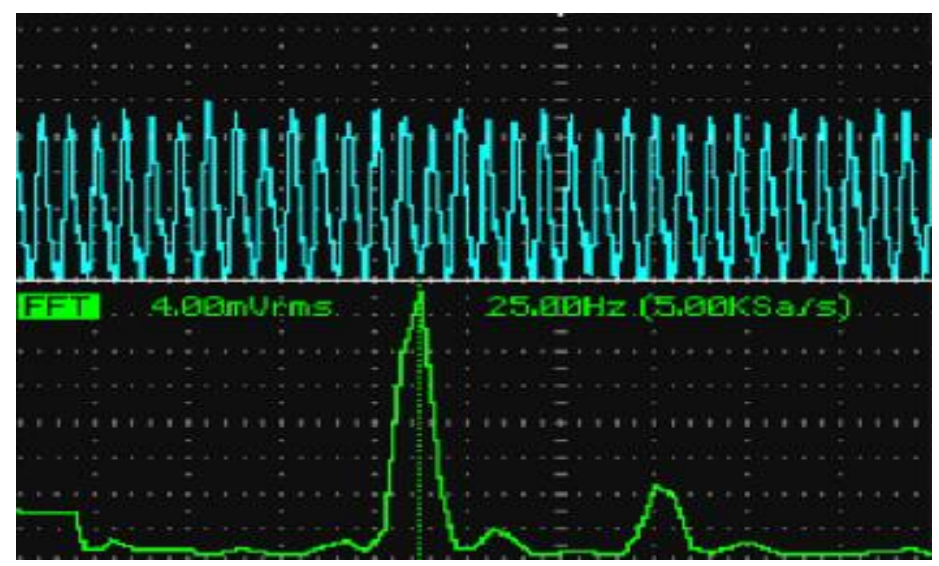

Fig. 10. Time domain and frequency spectrum (FFT) of experimental vibration signal of globe valve.

It is obvious that the vibration measurements will determine the frequency spectrum and the amplitude of vibration but does notsupply details about the source of shaking because the absolute value of vibration does not give a suitable knowledge about the flow behavior and vibration source inside the piping system. The first observation on the vibration data is that the behavior of experimental measured vibration signal is close to that obtained from 1-way numerical simulation than that obtained from 2way numerical simulation. Experimental tests show that the vibration signal was repeated with approximately constant frequency $(336 \mathrm{~Hz})$ and amplitude $(0.4 \mathrm{~mm})$ and there is one distinguish peak in frequency spectrum. Similarly, the 1-way simulation provides repeated vibration signal with some noise and frequency spectrum shows one distinguish peak. The vibration signal obtained from 2-way simulation has remarkable decay in amplitude and changing frequency until approaches to convergence state. In addition, the frequency spectrum shows more than one distinguish peak $(300 \mathrm{~Hz})$. This is an important observation because it confirms the validity of numerical simulation. The 2-way numerical simulation is an important issue when the structure undergoing large deflection vibration. This observation indicates that 1-way FSI simulation is sufficient to estimate the dynamic response of piping structure in current study where the vibration of valve can be considered as small deflection vibration. Different operation conditions, support type, material size, types, and boundary conditions of the same structure may be made 1-way FSI simulation not sufficient to 
predict the dynamic response (vibration). The second and most important observation on the vibration results that the response of valve to flow excitation is approximately repeated with amount of noise in the form of random vibration. This indicates that the dominant source of vibration can be water pulsation or water hammering or vortex shedding because all these sources provide a regular excitation wave. Water pulsation and water hammering are including pressure waves. Water pulsation included steady oscillation with small amplitude. This small amplitude can be developed and enlarge if the resonance conditions are realize while the water hammering is unsteady transient oscillations with large amplitude. The water hammering may occured in valves and pumps but it is related to fluid -structure interaction. The comparisons between the experimental vibration data and the numerical simulation results show that no significant FSI was established for current case study. This observation proves that the dominant source of vibration for valve is the water pulsation in addition to amount of water hammering. Now, it is a valuable to specify the source of water pulsation for flow in valves. By return to figures 6 and 7 , the main source of water pulsation is the vortex shedding and pressure difference between upstream and downstream of valve. Figure 7 shows many circulation regions which can be the source of vortex. The vortex shedding is a regular flow phenomenon can cause regular pressure wave which in turn excite the structure to vibrate. Figure 6 shows high reduction of pressure cross the valve section. The pressure reduction causes the high amount of turbulence energy transferred into the system and can be the source of associated random vibration (noise).In addition, the appearance of one distinguish peak in frequency spectrum for both experimental and 1way numerical simulation proves that the system may vibrates close to one natural mode of vibration. This phenomenon called lock-in where the frequency of vortex shedding coincides with one of the natural frequencies of the system. The other subsequent result from vibration data and flow characteristics that the vibration of valve depends on the flow path and the flow rate of water sincethe flow characteristics (velocity and pressure)distribution has strong dependency on the amount of valve enclosure (flow path) as shown in figures 6-7. Figure 11 shows the vibration measurement data (displacement signal) of globe valve measured at different flow rate cross the valve (enclosing percentage).

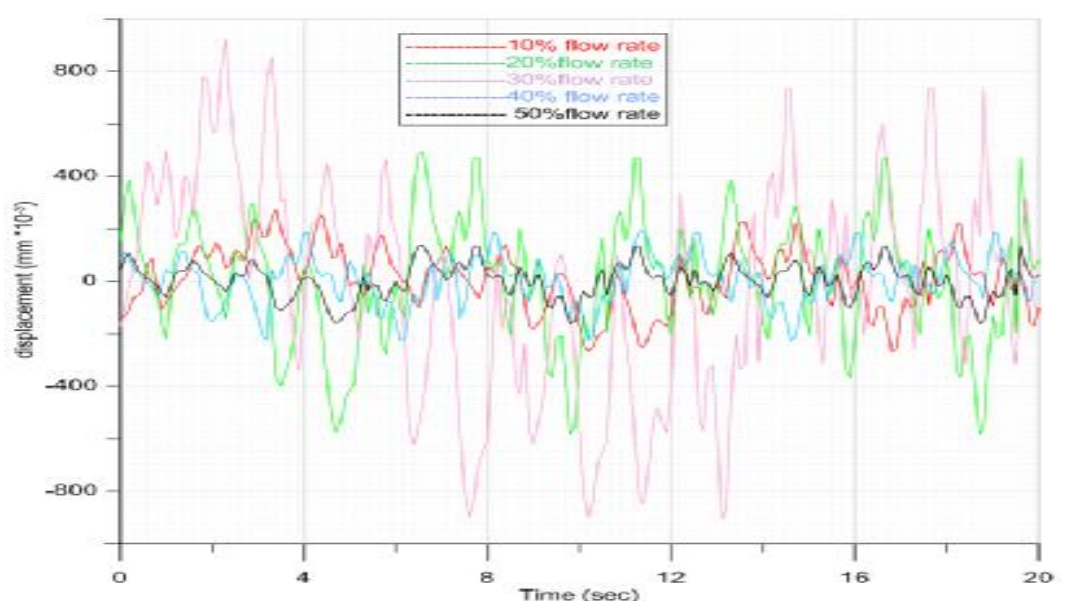

Fig. 11. Displacement measurement of globe valve at different flow rate cross the valve.

It is obvious from the figure that the vibration amplitude increases with increasing of flow rate to be maximum when the flow rate around $30 \%$ and then decreased until flow rate reaches to around $85 \%$ and the trends to be constant as shown in figure 12 . 


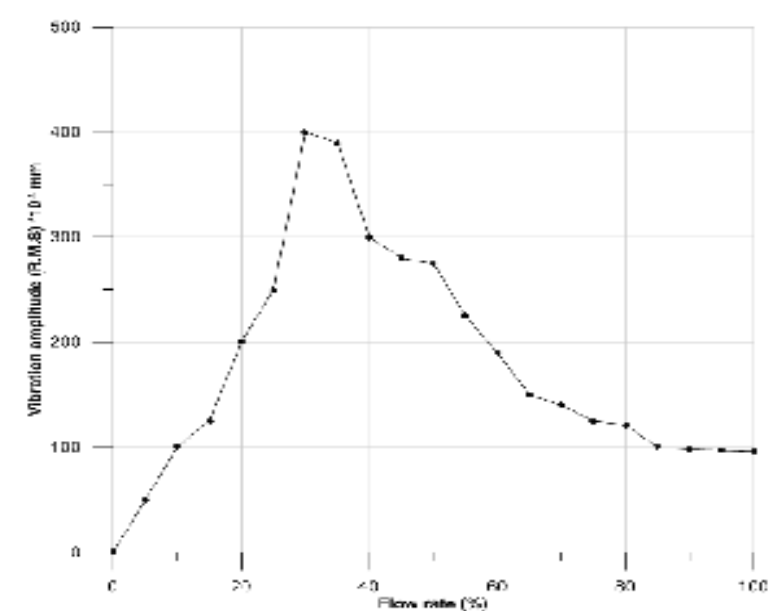

Fig. 12.Vibration amplitude of globe valve versus flow rate cross the valve. [Experimental data].

This behavior of vibration is related to flow characteristics as mentioned. Figure 7 shows that severe flow conditions (high pulsation and vortex shedding) between enclosure percentages $80-60 \%$ [flow rate $20-40 \%$ ]. This confirms the previous results. The dependency of vibration level on flow characteristics (flow phenomenon's, flow path, flow rate, pressure distribution, and velocity distribution) requires other experimental and numerical analysis on valve of different geometry.

\section{Conclusion}

1. Experimental tests show that the structural vibration signal was approximately repeated with approximately constant frequency $(336 \mathrm{~Hz})$ and amplitude with little noise signals. This can be shown from FFT of experimental data where one distinguish peak represent the dominant vibration frequency and many small peaks represent the noise.

2. There is one distinguish peak in frequency spectrum for experimental vibration measurement.

3. The behavior of experimental measured vibration signal of valve is close to that obtained from 1-way numerical simulation than that obtained from 2-way numerical simulation. Therefore, the 1-way FSI simulation is sufficient to estimate the dynamic response (vibration) of valve because it can be considered as small deflection vibration.

4. The dominant source of vibration of valves can be water pulsation or water hammering or vortex shedding because all these sources provide a regular excitation wave with approximately constant frequency at steady state of velocity and pressure.

5. The dominant source of vibration for globe valve established in current study is the water pulsation in addition to amount of water hammering

6. The main source of water pulsation in globe valve is the vortex shedding and pressure difference between upstream and downstream of valve.

7. The vibration of valve dependent on the flow path and the flow rate of water since the flow characteristics (velocity and pressure) distribution has strong dependency on the amount of valve enclosure (flow path)

8. The vibration amplitude increases with increasing of flow rate to be maximum when the flow rate around $30 \%$ and then decreased until flow rate reaches to around $85 \%$ and then trends to be constant

\section{Nomenclature}

\begin{tabular}{|c|c|}
\hline Symbol & Description \\
\hline $\mathrm{x}, \mathrm{y}$, and $\mathrm{z}$ & Cartesian coordinates. \\
\hline $\mathrm{u}, \mathrm{v}$, and $\mathrm{w}$ & $\begin{array}{l}\text { Velocity component in direction of } \mathrm{x} \text {, } \\
\mathrm{y} \text {, and } \mathrm{z} \text { respectively. }\end{array}$ \\
\hline $\mathrm{P}$ & Pressure $\left(\mathrm{N} / \mathrm{m}^{2}\right)$ \\
\hline$\rho$ & Fluid density $\left(\mathrm{kg} / \mathrm{m}^{3}\right)$ \\
\hline $\mathrm{g}$ & Gravitational acceleration $\left(\mathrm{m} / \mathrm{s}^{2}\right)$ \\
\hline$\mu$ & Viscosity (N.s/m²) \\
\hline$[\mathrm{M}]$ & Mass matrix $[\mathrm{kg}]$ \\
\hline [c] & Damping coefficient matrix $[\mathrm{kg} / \mathrm{s}]$ \\
\hline$[\mathrm{k}]$ & Stiffness matrix $\left[\mathrm{kg} / \mathrm{s}^{2}\right]$ \\
\hline$\delta, \dot{\delta}, \ddot{\delta}:$ & $\begin{array}{l}\text { Displacement, velocity and } \\
\text { acceleration of structural particle } \\
\text { respectively. }\end{array}$ \\
\hline
\end{tabular}

\section{References}

[1]N. H. Fletcher, "Autonomous vibration of simple pressure-controlled valves in gas flows", J. Acoustic. Soc. Am. 93(4), Pt. 1, April 1993.

[2] N.E. LIGTERINK, R. DE GROOT, H.J. SLOT , EMAD GHARAIBAH, "Flow induced vibration of subsea gas production systems caused by choke valves", SPE Annual Technical Conference and Exhibition held on 8-10 October 2012, San Antonio, Texas. Copyright 2012, Society of Petroleum Engineers. 
[3] Ravi Pankhania, Dhaval Patel and V.M. Bhojawala, "Free Vibration Analysis of a Motor Operated Gate Valve for Nuclear Application Using Finite Element Analysis", International Journal of Advanced Mechanical Engineering. ISSN 2250-3234 Volume 4, Number 3 (2014), pp. 349-356.

[4] Malik I. Al-Amayreh, Mohammad I. Kilani, Ahmed S. Al-Salaymeh, " Numerical Study of a Butterfly Valve for Vibration Analysis and Reduction" International Journal of Mechanical, Aerospace, Industrial, Mechatronic and Manufacturing Engineering Vol:8, No:12, 2014.

[5]ANSYS theory reference. Twelve edition, 2009, ANSYS Inc., South Point, Canonsburg,

[6]H. H. Hamad, "Dynamic response of solar collector for self-cleaning", Ph.D thesis, university of technology, 2012.
[7]C. Taylor and T. G. Hughes, "Finite Element Programming of the Navier-Stokes Equations", Bell and Bain Ltd., 1st Published, 1981.

[8] Sinan A. Ali " Study effect of Riblet Upstream Wing-Wall junction Numerically and Experimentally" ,M.Sc. thesis, Mechanical eng. Dept. University of Technology, 2011.

[9]Friedel Hartmann Casimir Katz "Structural Analysis with Finite Elements" SpringerVerlag Berlin Heidelberg 2007.

[10] Ihsan.A. Baqer, "Modeling of 3D Grasping of artificial hand under dynamic load", Ph.D thesis, University of technology, Baghdad, 2014. 


\section{بحث عملي وعددي على الاهتزاز الناتج عن الجريان في الصمامات

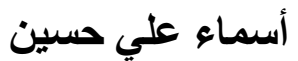

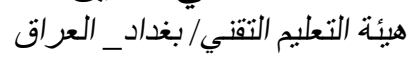 \\ البريد الالكتروني:yahoo.com}

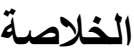

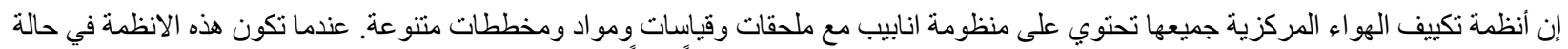

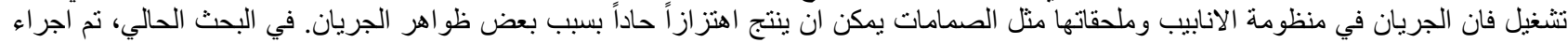

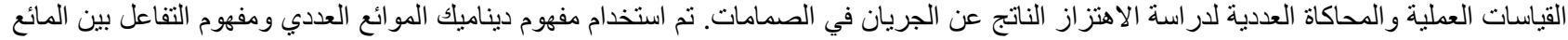

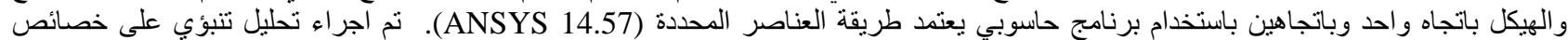

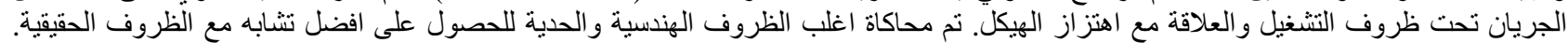

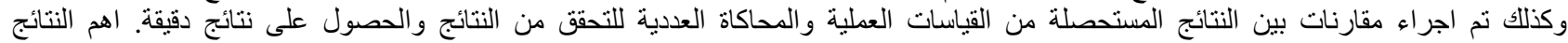

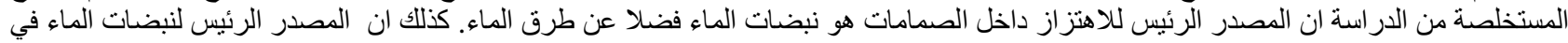

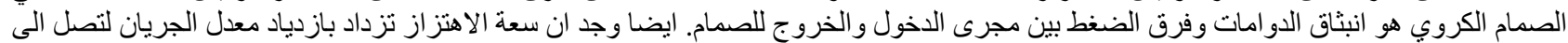

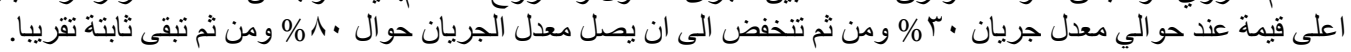

